\title{
ON CABLED KNOTS, DEHN SURGERY, AND LEFT-ORDERABLE FUNDAMENTAL GROUPS
}

\author{
Adam Clay and Liam Watson
}

\begin{abstract}
Previous work of the authors establishes a criterion on the fundamental group of a knot complement that determines when Dehn surgery on the knot will have a fundamental group that is not left-orderable [6]. We provide a refinement of this criterion by introducing the notion of a decayed knot; it is shown that Dehn surgery on decayed knots produces surgery manifolds that have non-left-orderable fundamental group for all sufficiently positive surgeries. As an application, we prove that sufficiently positive cables of decayed knots are always decayed knots. These results mirror properties of L-space surgeries in the context of Heegaard Floer homology.
\end{abstract}

\section{Introduction}

Definition 1.1. A group $G$ is left-orderable if there exists a partition of the group elements

$$
G=\mathcal{P} \sqcup\{1\} \sqcup \mathcal{P}^{-1}
$$

satisfying $\mathcal{P} \cdot \mathcal{P} \subseteq \mathcal{P}$ and $\mathcal{P} \neq \emptyset$. The subset $\mathcal{P}$ is called a positive cone.

This is equivalent to $G$ admitting a left-invariant strict total ordering. For background on left-orderable groups relevant to this paper see $[2,6]$; a standard reference for the theory of left-orderable groups is [12]. As established by Boyer et al. [2] (compare [11]), the fundamental group $\pi_{1}(K)$ of the complement of a knot $K$ in $S^{3}$ is always left-orderable. Indeed, this follows from the fact that any compact, connected, irreducible, orientable three-manifold with positive first Betti number has left-orderable fundamental group [2, Theorem 1.1]. However, the question of left-orderability for fundamental groups of rational homology three-spheres is considerably more subtle (see $[2,6]$ ) and seems closely tied to certain codimension one structures on the threemanifold (see $[2,3,17]$ ). Continuing along the lines of $[6]$ this paper focuses on Dehn surgery, an operation on knots that produces rational homology three-spheres. We recall this construction in order to fix notation and conventions.

For any knot $K$ in $S^{3}$ there is a preferred generating set for the peripheral subgroup $\mathbb{Z} \oplus \mathbb{Z} \subset \pi_{1}(K)$ provided by the knot meridian $\mu$ and the Seifert longitude $\lambda$. The latter is uniquely determined (up to orientation) by the existence of a Seifert surface for $K$. We orient $\mu$ so that it links positively with $K$, and orient $\lambda$ so that $\mu \cdot \lambda=1$. For any rational number $r$ with reduced form $\frac{p}{q}$ we denote the peripheral element $\mu^{p} \lambda^{q}$ by $\alpha_{r}$. At the level of the fundamental group, the result of Dehn surgery along $\alpha_{r}$ is summarized by the short exact sequence

$$
1 \rightarrow\left\langle\left\langle\alpha_{r}\right\rangle\right\rangle \rightarrow \pi_{1}(K) \rightarrow \pi_{1}\left(S_{r}^{3}(K)\right) \rightarrow 1 .
$$

Received by the editors April 14, 2011. 
Here $\left\langle\left\langle\alpha_{r}\right\rangle\right\rangle$ denotes the normal closure of $\alpha_{r}$, and $S_{r}^{3}(K)$ is the three-manifold obtained by attaching a solid torus to the boundary of $S^{3} \backslash \nu(K)$, sending the meridian of the torus to a simple closed curve representing the class

$$
\left[\alpha_{r}\right] \in H_{1}\left(\partial\left(S^{3} \backslash \nu(K)\right) ; \mathbb{Z}\right) /\{ \pm 1\} .
$$

We will blur the distinction between $\alpha_{r}$ as an element of the fundamental group or as a primitive class in the (projective) first homology of the boundary, and refer to these peripheral elements as slopes.

While many examples of rational homology three-spheres have left-orderable fundamental group [2], there exist infinite families of knots for which sufficiently positive Dehn surgery (that is, along a slope parameterized by a suitable large rational number) yields a manifold with non-left-orderable fundamental group [6]. To make this precise, consider the set of slopes

$$
\mathcal{S}_{r}=\left\{\alpha_{r^{\prime}} \mid r^{\prime} \geq r\right\}
$$

for some fixed rational $r$.

Definition 1.2. A non-trivial knot $K$ in $S^{3}$ is called $r$-decayed if, for any positive cone $\mathcal{P}$ in $\pi_{1}(K)$, either $\mathcal{P} \cap \mathcal{S}_{r}=\mathcal{S}_{r}$ or $\mathcal{P} \cap \mathcal{S}_{r}=\emptyset$.

The existence of decayed knots is established in [6]. For example, the torus knot $T_{p, q}$ is $(p q-1)$-decayed (for $\left.p, q>0\right)$, and the $(-2,3, q)$-pretzel knot is $(10+q)$ decayed for odd $q \geq 5$ (see Theorem 2.1). Our interest in this property stems from the following:

Theorem 1.1. If $K$ is $r$-decayed then $\pi_{1}\left(S_{r^{\prime}}^{3}(K)\right)$ is not left-orderable for all $r^{\prime} \geq r$.

As a result, it is not restrictive to assume that $r$ is a positive rational number since $\pi_{1}\left(S_{0}^{3}(K)\right)$ is always left-orderable [2]. Notice however that it is not immediately clear how Theorem 1.1 might be applied in practice, as there is no obvious method for checking when a knot is $r$-decayed. For this reason, in Section 2 we describe an equivalent formulation of $r$-decay whose statement is more technical, but easier to verify, than the definition. Together with the proof of Theorem 1.1, the results of Section 2 provide a useful refinement of the ideas from [6].

Results connecting left-orderability and Dehn surgery may be expected to mirror similar results relating to L-spaces, since there is no known example of an L-space with left-orderable fundamental group, while many L-spaces have fundamental group that is not left-orderable, (see $[1,5,6,16,21]$ ). Recall that an L-space is a rational homology sphere with Heegaard Floer homology that is as simple as possible, in the sense that $r k \widehat{\mathrm{HF}}(Y)=\left|H_{1}(Y ; \mathbb{Z})\right|$ (see $\left.[15]\right)$. Theorem 1.1 mirrors a fundamental property of knots admitting L-space surgeries: if $S_{n}^{3}(K)$ is an L-space, then $S_{r}^{3}(K)$ is an L-space as well for any $r \geq n$.

In the interest of further investigating left-orderability of fundamental groups of 3-manifolds along the lines of [6], we consider the behaviour of Dehn surgery on cables of $r$-decayed knots (for necessary background, see Section 3 ). Denoting the $(p, q)$-cable of the knot $K$ as $C_{p, q}(K)$, the main theorem of this article is:

Theorem 1.2. If $K$ is $r$-decayed then $C_{p, q}(K)$ is pq-decayed whenever $\frac{q}{p}>r$. 
The proof of Theorem 1.2 is contained in Section 3. Notice that combining Theorem 2.1 and Theorem 1.2 provides a rather large class of knots for which sufficiently positive surgery yields a non-left-orderable fundamental group.

Dehn surgery on cabled knots and non-left-orderability of the resulting fundamental groups may again be viewed in the context of Heegaard Floer homology. Referring to knots admitting L-space surgeries as L-space knots, Hedden proves:

Theorem 1.3. [9, Theorem 1.10] If $K$ is an L-space knot then $C_{p, q}(K)$ is an L-space knot whenever $\frac{q}{p} \geq 2 g(K)-1$.

Here, the quantity $g(K)$ is the Seifert genus of $K$. Note that the converse of this statement has been recently established by Hom [10].

In order to assess the strength of Theorem 1.2, it is natural to ask when Dehn surgery on a cable knot yields a manifold that has left-orderable fundamental group. It turns out that, in the case that $K$ is $r$-decayed, Theorem 1.2 is close to describing all possible non-left-orderable surgeries on a cable knot $C_{p, q}(K)$, in the following sense:

Theorem 1.4. Suppose that $C$ is the $(p, q)$-cable of some knot. If $r \in \mathbb{Q}$ satisfies $r<p q-p-q$, then $\pi_{1}\left(S_{r}^{3}(C)\right)$ is left-orderable.

This result is a special case of a more general observation pertaining to satellite knots that is discussed in Section 4. Notice that Theorem 6 makes no reference to the original knot being $r$-decayed. However, restricted to $r$-decayed knots, Theorem 1.2 and Theorem 1.4 combine to produce an interval of surgery coefficients for which the left-orderability of the associated quotient is not determined. More precisely:

Question 1.1. If $K$ is $r$-decayed and $C$ is a $(p, q)$-cable of $K$ with $\frac{q}{p}>r$, can Theorem 1.2 and Theorem 1.4 be sharpened to determine when $\pi_{1}\left(S_{r^{\prime}}^{3}(C)\right)$ is leftorderable for $r^{\prime}$ satisfying $p q-p-q<r^{\prime}<p q$ ?

\section{A practical reformulation of Theorem 1.1}

We begin with a reformulation of $r$-decay that will be essential in connecting this work with the results of [6]. This will require the following lemma:

Lemma 2.1. Let $G$ be a left-orderable group containing elements $g, h$. If $g \in \mathcal{P}$ implies $h \in \mathcal{P}$ for every positive cone $\mathcal{P}$, then $g \in \mathcal{P}$ if and only if $h \in \mathcal{P}$.

Proof. We need only show the converse, namely $h \in \mathcal{P}$ implies $g \in \mathcal{P}$ for every positive cone $\mathcal{P} \subset G$. For a contradiction, suppose this is not the case, so there exists a positive cone such that $h \in \mathcal{P}$ and $g \notin \mathcal{P}$. Consider the positive cone $\mathcal{Q}=\mathcal{P}^{-1}$, defining the reverse ordering of $G$. This gives $g \in \mathcal{Q}$ and $h \notin \mathcal{Q}$, contradicting our assumption.

Proposition 2.1. A knot $K$ is $r$-decayed if and only if for every positive cone $\mathcal{P} \subset$ $\pi_{1}(K)$ there exists a strictly increasing sequence of positive rational numbers $\left\{r_{i}\right\}$ with $r_{i} \rightarrow \infty$ satisfying

(1) $r=r_{0}$, and

(2) $\alpha_{r} \in \mathcal{P}$ implies $\alpha_{r_{i}} \in \mathcal{P}$ for all $i$. 
Proof. Suppose that $K$ is $r$-decayed, and let $\mathcal{P}$ be any positive cone. Choose a strictly increasing sequence of rational numbers $\left\{r_{i}\right\}$ with $r_{0}=r$ and $r_{i} \rightarrow \infty$. Whenever $\alpha_{r}=\alpha_{r_{0}} \in \mathcal{P}$ we have $\mathcal{S}_{r} \cap \mathcal{P} \neq \emptyset$, so that $\mathcal{S}_{r} \cap \mathcal{P}=\mathcal{S}_{r}$ since $K$ is $r$-decayed. It follows that $\alpha_{r_{i}} \in \mathcal{S}_{r} \subset \mathcal{P}$ for all $i$.

To prove the converse, let $\mathcal{P}$ be a positive cone for $\pi_{1}(K)$. Fix a strictly increasing sequence $\left\{r_{i}\right\}$ of rational numbers limiting to infinity and satisfying (1) and (2). Suppose that $\alpha_{r} \in \mathcal{P}$, then by assumption $\alpha_{r_{i}} \in \mathcal{P}$ for all $i>0$.

Now suppose that $\mu^{m} \lambda^{n}$ is an element of $\mathcal{S}_{r}$. Choose $r_{i}, r_{i+1}$ with corresponding reduced forms $\frac{p_{i}}{q_{i}}, \frac{p_{i+1}}{q_{i+1}}$ such that $r_{i}<\frac{m}{n}<r_{i+1}$. By solving

$$
\begin{aligned}
q_{i} a+q_{i+1} b & =c n, \\
p_{i} a+p_{i+1} b & =c m,
\end{aligned}
$$

we can find positive integers $a, b$ and $c$ such that $\left(\mu^{p_{i}} \lambda^{q_{i}}\right)^{a}\left(\mu^{p_{i+1}} \lambda^{q_{i+1}}\right)^{b}=\left(\mu^{m} \lambda^{n}\right)^{c}$. Explicitly, Cramer's rule gives

$$
a=\left|\begin{array}{cc}
n & q_{i+1} \\
m & p_{i+1}
\end{array}\right|, \quad b=\left|\begin{array}{cc}
q_{i} & n \\
p_{i} & m
\end{array}\right|, \quad c=\left|\begin{array}{cc}
q_{i} & q_{i+1} \\
p_{i} & p_{i+1}
\end{array}\right| ;
$$

note that all these quantities are positive because of our restriction $r_{i}<\frac{m}{n}<r_{i+1}$ (compare [6, Lemma 17]) . This shows that $\mu^{m} \lambda^{n}$ is positive, since its $c$ th power is expressed as a product of positive elements. Hence $\mathcal{S}_{r} \cap \mathcal{P}=\mathcal{S}_{r}$.

This establishes the implication $\alpha_{r} \in \mathcal{P} \Rightarrow \mathcal{S}_{r} \subset \mathcal{P}$ for every positive cone $\mathcal{P}$. By Lemma 2.1, this is equivalent to $\mathcal{S}_{r} \cap \mathcal{P}=\mathcal{S}_{r}$ or $\mathcal{S}_{r} \cap \mathcal{P}=\emptyset$ for every positive cone $\mathcal{P}$, so that $K$ is $r$-decayed.

Remark 2.1. In practice, it is often more natural to establish $\alpha_{r} \in \mathcal{P}$ implies $\alpha_{r_{i}}^{w_{i}} \in \mathcal{P}$ for all $i$, where $w_{i} \in \mathbb{N}$ (see in particular the proofs of Lemmas 3.2 and 3.3). This situation arises when one constructs (for a given positive cone $\mathcal{P}$ ) a sequence of unreduced rationals $\left\{r_{i}\right\}=\left\{\frac{p_{i}}{q_{i}}\right\}$ for which $\operatorname{gcd}\left(p_{i}, q_{i}\right)=w_{i} \geq 1$, and $\mu^{p_{0}} \lambda^{q_{0}} \in \mathcal{P}$ implies $\mu^{p_{i}} \lambda^{q_{i}} \in \mathcal{P}$ for all $i$. Notice that the implication $\alpha_{r} \in \mathcal{P}$ implies $\alpha_{r_{i}}^{w_{i}} \in \mathcal{P}$ still allows us to apply Proposition 2.1, since $\alpha_{r_{i}}^{w_{i}} \in \mathcal{P}$ if and only if $\alpha_{r_{i}} \in \mathcal{P}$ (this simple observation holds in any left-orderable group). Ultimately, this results in more flexibility in selecting the sequence $\left\{r_{i}\right\}$.

The equivalence established in Proposition 2.1 shows that all examples considered in [6] are $r$-decayed for certain $r$, as [6, Corollary 11] is a special case of Proposition 2.1.

Theorem 2.1. [6, Theorems 24, 28 and 30]

(1) The $(p, q)$-torus knot is $(p q-1)$-decayed for all positive, relatively prime pairs of integers $p, q$.

(2) The $(-2,3, q)$-pretzel knot is $(10+q)$-decayed for all odd $q \geq 5$.

(3) The $(3, q)$-torus knot with one positive full twist added along two strands is $(3 q+2)$-decayed, for all positive $q$ congruent to 2 modulo 3.

Proof. We consider the case of $K_{q}$, the $(-2,3, q)$-pretzel knot with $q \geq 5$ odd, the other cases are similar. Set $r=10+q$, and $r_{i}=r+i$. It is shown in [6] that for every positive cone $\mathcal{P}$ in $\pi_{1}\left(K_{q}\right)$, the implication $\alpha_{r} \in \mathcal{P} \Rightarrow \alpha_{r_{i}} \in \mathcal{P}$ holds for all $i \geq 0$. This means that for every left-ordering of $\pi_{1}\left(K_{q}\right)$, the integer sequence $\left\{r_{i}\right\}$ satisfies the properties required by Proposition 2.1, and we conclude that $K_{q}$ is $r$-decayed. 
Note that the above proof illustrates some particularly special behaviour, as the rational sequences $\left\{r_{i}\right\}$ required by Proposition 2.1 (which a priori may be different for each left-ordering) are replaced by a single integer sequence sufficient for every left-ordering. Thus, Proposition 2.1 provides a more workable method (than used previously) for checking when a knot has surgeries that yield a non-left-orderable fundamental group. Combined with the material established in [6, Section 2], we provide a short proof of Theorem 1.1.

Proof of Theorem 1.1. For contradiction, assume that $\pi_{1}\left(S_{r^{\prime}}^{3}(K)\right)$ is left-orderable for some $r^{\prime} \geq r$, and consider the short exact sequence

$$
1 \rightarrow\left\langle\left\langle\alpha_{r^{\prime}}\right\rangle\right\rangle \stackrel{i}{\rightarrow} \pi_{1}(K) \stackrel{f}{\rightarrow} \pi_{1}\left(S_{r^{\prime}}^{3}(K)\right) \rightarrow 1,
$$

as defined in the introduction. Let $\mu, \lambda \in \pi_{1}(K)$ denote the meridian and longitude. Since $\pi_{1}\left(S_{r^{\prime}}^{3}(K)\right)$ is left-orderable, $\left\langle\left\langle\alpha_{r^{\prime}}\right\rangle\right\rangle \cap\langle\mu, \lambda\rangle=\left\langle\alpha_{r^{\prime}}\right\rangle$ (see [6, Proof of Proposition $20])$. In particular, if we fix an arbitrary rational number $s_{0}>r^{\prime}$, then $f\left(\alpha_{s_{0}}\right) \neq 1$. Thus, we may choose a positive cone $\mathcal{Q}$ in $\pi_{1}\left(S_{r^{\prime}}^{3}(K)\right)$ that contains $f\left(\alpha_{s_{0}}\right)$. Next, choose a positive cone $\mathcal{Q}^{\prime} \subset\left\langle\left\langle\alpha_{r^{\prime}}\right\rangle\right\rangle$ not containing $\alpha_{r^{\prime}}$, and define a positive cone $\mathcal{P} \subset \pi_{1}(K)$ by

$$
\mathcal{P}=i\left(\mathcal{Q}^{\prime}\right) \sqcup f^{-1}(\mathcal{Q}) .
$$

Note that $\alpha_{r^{\prime}} \notin \mathcal{P}$, and $\alpha_{s_{0}} \in \mathcal{P}$.

This is a standard construction for creating a left-ordering of a group using a short exact sequence, here the result is a left-ordering of $\pi_{1}(K)$ with positive cone $\mathcal{P}$, relative to which the subgroup $\left\langle\left\langle\alpha_{r^{\prime}}\right\rangle\right\rangle$ is convex. Because $\left\langle\left\langle\alpha_{r^{\prime}}\right\rangle\right\rangle$ is convex, the intersection $\left\langle\left\langle\alpha_{r^{\prime}}\right\rangle\right\rangle \cap\langle\mu, \lambda\rangle=\left\langle\alpha_{r^{\prime}}\right\rangle$ is convex in the restriction ordering of $\langle\mu, \lambda\rangle$. Therefore, [6, Proposition 18] shows that all slopes $\alpha_{s}$ with $s>r^{\prime}$ must have the same sign. In particular, since $\alpha_{s_{0}}$ is positive it follows that all slopes $\alpha_{s}$ with $s>r^{\prime}$ are positive, so that

$$
Q \cap \mathcal{S}_{r}=\left\{\alpha_{s} \mid s>r^{\prime}\right\}
$$

Therefore, $K$ is not $r$-decayed.

We remark that there is a more geometric argument establishing Theorem 1.1, that relies upon an understanding of the topology of the space of left-orderings of $\mathbb{Z} \oplus \mathbb{Z}$ (see [18, Section 3] and [4, Chapter 6]). Roughly, every left-ordering of the knot group $\pi_{1}(K)$ restricts to a left-ordering of the peripheral subgroup that defines a line in $\mathbb{Z} \oplus \mathbb{Z}$, with all positive elements of $\mathbb{Z} \oplus \mathbb{Z}$ on one side of the line, and all the negative elements on the other side. As a result, given two rationals $r_{1}<r_{2}$ corresponding to slopes $\alpha_{r_{1}}$ and $\alpha_{r_{2}}$ that have the same sign in every left-ordering, no left-ordering can restrict to an ordering of the peripheral subgroup with corresponding slope $s$ between $r_{1}$ and $r_{2}$. The proof of Theorem 1.1 then follows from checking that whenever $\pi_{1}\left(S_{r^{\prime}}^{3}(K)\right)$ is left-orderable, we can define a left-ordering of $\pi_{1}(K)$ that restricts to yield a line of slope $r^{\prime}$ in the peripheral subgroup (compare [6, Proof of Theorem 9]).

\section{The proof of Theorem $\mathbf{1 . 2}$}

We recall the construction of a cabled knot in order to fix notation. Consider the $(p, q)$-torus knot $T_{p, q}$, where $p, q>0$ are relatively prime. As the closure of a $p$-strand braid, this knot may be naturally viewed in a solid torus $T$ by removing a tubular 
neighbourhood of the braid axis. The complement of $T_{p, q}$ in $T$ is referred to as a $(p, q)$-cable space. Now given any knot $K$ in $S^{3}$, the cable knot $C_{p, q}(K)$ is obtained by identifying the boundary of $T$ with the boundary of $S^{3} \backslash \nu(K)$, identifying the longitude of $T$ with the longitude $\lambda$ of $K$. We will denote this cable knot by $C$ whenever this simplified notation does not cause confusion.

The knot group $\pi_{1}(C)$ may be calculate via the Seifert-Van Kampen Theorem, by viewing the complement $S^{3} \backslash \nu(C)$ as the identification of the boundaries of $S^{3} \backslash \nu(K)$ and a solid torus $D^{2} \times S^{1}$ along an essential annulus with core curve given by the slope $\mu^{q} \lambda^{p}$. If $\pi_{1}\left(D^{2} \times S^{1}\right)=\langle t\rangle$ then this gives rise to a natural amalgamated product

$$
\pi_{1}(C) \cong \pi_{1}(K) *_{\mu^{q} \lambda^{p}=t^{p}} \mathbb{Z} .
$$

Consulting [6, Section 3], the meridian and longitude for $C$ may be calculated as

$$
\mu_{C}=\mu^{u} \lambda^{v} t^{-v} \quad \text { and } \quad \lambda_{C}=\mu_{C}^{-p q} t^{p}
$$

where $u$ and $v$ are positive integers satisfying $p u-q v=1$ (compare [20, Proof of Theorem 3.1]).

Suppose that the knot $K$ is $r$-decayed, and choose cabling coefficients $p$ and $q$ so that $q / p>r$. To begin, we choose a positive cone $\mathcal{P} \subset \pi_{1}(C)$ and assume, without loss of generality, that $\mu_{C}^{p q} \lambda=t^{p}$ is positive. This means that $t^{p}=\mu^{q} \lambda^{p} \in \mathcal{P}$, so every element $\mu^{m} \lambda^{n}$ is positive whenever $m / n>r$, since $K$ is $r$-decayed. To see this, note that the inclusion $\pi_{1}(K) \subset \pi_{1}(C)$ means that every left-ordering of $\pi_{1}(C)$ induces a left-ordering of $\pi_{1}(K)$ by restriction. In particular, if two elements of the subgroup $\langle\mu, \lambda\rangle \subset \pi_{1}(C)$ have opposite signs in a left-ordering of $\pi_{1}(C)$, then they will also have opposite signs in the induced left-ordering of $\pi_{1}(K)$. As a result we can use $r$-decay of the subgroup $\pi_{1}(K)$ as a property of left-orderings of the larger group $\pi_{1}(C)$.

Our method of proof will be to check that the cable is $p q$-decayed by using the equivalence from Proposition 2.1. In particular, we will show that for the given positive cone $\mathcal{P} \subset \pi_{1}(C)$ there exists an unbounded sequence of increasing rationals $\left\{r_{i}\right\}$ with $r_{0}=p q$, such that our assumption $\alpha_{p q}=\mu_{C}^{p q} \lambda_{C} \in \mathcal{P}$ implies $\alpha_{r_{i}} \in \mathcal{P}$ for all $i>0$.

First consider the case when $\mu_{C}$ is positive in the left-ordering defined by $\mathcal{P}$. Here, $\mu_{C}^{p q+N} \lambda_{C}$ is positive for $N \geq 0$, as it is a product of positive elements. Therefore in this case it suffices to choose $r_{i}=p q+i$ for all $i \geq 0$.

For the remainder of the proof, we assume that $\mu_{C}$ is negative. For repeated use below, we also observe the crucial identity

$$
\left(t^{-v}\right)^{p}\left(\mu^{u} \lambda^{v}\right)^{p}=\left(t^{p}\right)^{-v} \mu^{u p} \lambda^{v p}=\mu^{-q v} \lambda^{-p v} \mu^{u p} \lambda^{v p}=\mu^{p v-q u}=\mu,
$$

and recall that $t^{p}$ commutes with $\mu, \lambda, \mu_{C}$, and $\lambda_{C}$. Therefore, we also have

$$
\left(\mu^{u} \lambda^{v}\right)^{p}\left(t^{-v}\right)^{p}=\left(t^{-v}\right)^{p}\left(\mu^{u} \lambda^{v}\right)^{p}=\mu .
$$

Let $k$ be an arbitrary non-negative integer, and consider the element

$$
\mu^{-k}\left(t^{-v} \mu^{u} \lambda^{v}\right) \mu^{k} .
$$

If this element is positive for some $k$, then the required sequence is provided by Lemma 3.2 (proved below). Therefore, we may assume that

$$
\mu^{-k}\left(t^{-v} \mu^{u} \lambda^{v}\right) \mu^{k} \notin \mathcal{P},
$$

for all $k$. 
Similarly, for $k$ a non-negative integer, we consider

$$
\left(\mu^{-k} t^{-v} \mu^{k}\right)^{p-1}\left(\mu^{u} \lambda^{v}\right)^{p-1}
$$

If this element is positive for some non-negative $k$, then we can create the required sequence using Lemma 3.3 (proved below). Therefore, we may assume that

$$
\left(\mu^{-k} t^{-v} \mu^{k}\right)^{p-1}\left(\mu^{u} \lambda^{v}\right)^{p-1} \notin \mathcal{P},
$$

for all $k \geq 0$.

Observe that

$$
\left(\mu^{-k} t^{-v} \mu^{k}\right)^{p-1}\left(\mu^{u} \lambda^{v}\right)^{p-1}=\left(\mu^{-k} t^{v} \mu^{k}\right)\left(\mu^{-k} t^{-v p} \mu^{k}\right)\left(\mu^{u p-u} \lambda^{v p-v}\right),
$$

which, recalling that $t^{p}$ commutes with the elements $\mu$ and $\lambda$, simplifies to give

$$
\left(\mu^{-k} t^{v} \mu^{k}\right)\left(\mu^{-u} \lambda^{-v}\right) t^{-v p} \mu^{u p} \lambda^{v p}=\left(\mu^{-k} t^{v} \mu^{k}\right)\left(\mu^{-u} \lambda^{-v}\right) \mu=\mu^{-k} t^{v} \lambda^{-v} \mu^{-u} \mu^{k+1} \notin \mathcal{P}
$$

for all $k \geq 0$. Taking inverses yields

$$
\mu^{-k-1} \mu^{u} \lambda^{v} t^{-v} \mu^{k}=\mu^{-k-1} \mu_{C} \mu^{k} \in \mathcal{P} \text {. }
$$

For the following lemmas, let $>$ denote the left-ordering defined by the positive cone $\mathcal{P}$, so that $h>g$ whenever $g^{-1} h \in \mathcal{P}$. We can then calculate:

Lemma 3.1. If $\mu^{-k-1} \mu_{C} \mu^{k} \in \mathcal{P}$ holds for all $k \geq 0$, then $\mu^{N+q} \lambda^{p}$ must be positive for all $N \geq 0$.

Proof. Since $\mu^{-k-1} \mu_{C} \mu^{k}>1$, left-multiplying by $\mu^{k+1}$ gives $\mu_{C} \mu^{k}>\mu^{k+1}$ for all $k \geq 0$. Setting $k=0$ we obtain $\mu_{C}>\mu$, so that left-multiplying by $\mu_{C}$ gives rise to

$$
\mu_{C}^{2}>\mu_{C} \mu \text {. }
$$

By setting $k=1$, we get

$$
\mu_{C} \mu>\mu^{2}
$$

which combines with the previous expression to give $\mu_{C}^{2}>\mu^{2}$. Continuing in this manner, we obtain $\mu_{C}^{N}>\mu^{N}$ for all $N \geq 0$. Left-multiplying by $t^{p}$, it follows that

$$
\mu_{C}^{N+p q} \lambda_{C}=\mu_{C}^{N} t^{p}>\mu^{N} t^{p}=\mu^{N+q} \lambda^{p}>1,
$$

where the final inequality follows from the fact that $(N+q) / p>q / p>r$ and $K$ is $r$-decayed.

As a result, when (3.1) and (3.2) hold we may choose the sequence of rationals $r_{i}=p q+i$ for all $i \geq 0$, and the requirements of Proposition 2.1 are met.

To conclude the proof, we establish Lemmas 3.2 and 3.3.

Lemma 3.2. If $\mu^{-k}\left(t^{-v} \mu^{u} \lambda^{v}\right) \mu^{k} \in \mathcal{P}$ for some $k \geq 0$, then there exists a sequence of rationals $\left\{r_{i}\right\}$ with $r_{0}=p q$ such that $\alpha_{r_{i}}>1$ for all $i$.

Proof. For $N \geq 0$, we rewrite $\mu_{C}^{N}$ as

$$
\mu_{C}^{N}=\mu^{u} \lambda^{v} \mu^{k}\left(\mu^{-k}\left(t^{-v} \mu^{u} \lambda^{v}\right)^{N} \mu^{k}\right) \mu^{-u-k} \lambda^{-v} .
$$

Fix a positive integer $s$ that is large enough so that $(s q-u-k) /(s p-v)>r$, this is possible because $q / p>r$. Next, the product $\mu_{C}^{N+p q s} \lambda_{C}^{s}=\mu_{C}^{N} t^{p s}$ becomes $\mu_{C}^{N} \mu^{s q} \lambda^{s p}$, which is equal to

$$
\mu^{u+k} \lambda^{v}\left(\mu^{-k}\left(t^{-v} \mu^{u} \lambda^{v}\right)^{N} \mu^{k}\right)\left(\mu^{q s-u-k} \lambda^{p s-v}\right) .
$$


This is a product of positive elements, because:

(a) $\mu^{u+k} \lambda^{v}>1$ because $(u+k) / v>u / v>q / p>r$ (recalling that $\left.p u-q v=1\right)$, and

(b) $\mu^{q s-u-k} \lambda^{p s-v}>1$ because $(s q-u-k) /(s p-v)>r$,

while the quantity $\mu^{-k}\left(t^{-v} \mu^{u} \lambda^{v}\right)^{N} \mu^{k}$ is positive by assumption. Therefore, in this case we choose our sequence of rationals to be

$$
r_{i}=\frac{p q s+i}{s}
$$

for $i \geq 0$, this guarantees that the associated slopes $\alpha_{r_{i}}$ are positive in the given left-ordering.

Lemma 3.3. If $\mu^{-k}\left(t^{-v} \mu^{u} \lambda^{v}\right) \mu^{k} \notin \mathcal{P}$ for all $k \geq 0$, and $\left(\mu^{-k} t^{-v} \mu^{k}\right)^{p-1}\left(\mu^{u} \lambda^{v}\right)^{p-1} \in \mathcal{P}$ for some $k \geq 0$, then there exists a sequence of rationals $\left\{r_{i}\right\}$ with $r_{0}=p q$ such that $\alpha_{r_{i}}>1$ for all $i$.

Proof. Fix $k \geq 0$ such that $\mu^{-k}\left(t^{-v} \mu^{u} \lambda^{v}\right) \mu^{k}<1$ and $\left(\mu^{-k} t^{-v} \mu^{k}\right)^{p-1}\left(\mu^{u} \lambda^{v}\right)^{p-1}>1$, and let $n$ be the smallest positive integer such that

$$
\left(\mu^{-k} t^{-v} \mu^{k}\right)^{n}\left(\mu^{u} \lambda^{v}\right)^{n}>1
$$

and

$$
\left(\mu^{-k} t^{-v} \mu^{k}\right)^{n-1}\left(\mu^{u} \lambda^{v}\right)^{n-1}<1,
$$

note that $1<n \leq p-1$ (If we take $n=p$, then the equality $\left(t^{-v}\right)^{p}\left(\mu^{u} \lambda^{v}\right)^{p}=\mu$ reduces the first expression to the identity, so $n=p$ is not possible). Note that we may rearrange these two expressions, so that

$$
\mu^{-k} t^{-v n}\left(\mu^{u} \lambda^{v}\right)^{n} \mu^{k}>1
$$

and

$$
\mu^{-k}\left(\mu^{u} \lambda^{v}\right)^{1-n} t^{-v(1-n)} \mu^{k}>1 .
$$

Then, for $N \geq 1$, we can rewrite the expression for $\mu_{C}^{N}$ as follows:

$\mu^{u+k} \lambda^{v}\left(\mu^{-k} t^{-v(1-n)} \mu^{k}\right)\left[\left(\mu^{-k} t^{-v n}\left(\mu^{u} \lambda^{v}\right)^{n} \mu^{k}\right) \mu^{-k}\left(\mu^{u} \lambda^{v}\right)^{1-n} t^{-v(1-n)} \mu^{k}\right]^{N-1} \mu^{-k} t^{-v n}$.

In the above expression, the quantity inside the square brackets is a product of positive elements. Denote this quantity by $P$. Choose an integer $s$ such that $(q s-k) / p s>r$. Then considering the slope $\mu_{C}^{N+p q(v+s)} \lambda_{C}^{v+s}=\mu_{C}^{N} t^{p(v+s)}$, we find

$$
\mu_{C}^{N} t^{p(v+s)}=\mu^{u+k} \lambda^{v}\left(\mu^{-k} t^{-v(1-n)} \mu^{k}\right) P^{N-1} \mu^{q s-k} \lambda^{p s} t^{p v-v n} .
$$

This is a product of positive elements, because:

(a) $\mu^{u+k} \lambda^{v}>1$, since $(u+k) / v>q / p>r$ (as before).

(b) $\mu^{-k} t^{-v(1-n)} \mu^{k}>1$, because if we consider its $p$ th power, we can use the fact that $t^{p}$ commutes with all peripheral elements so that

$$
\left(\mu^{-k} t^{-v(1-n)} \mu^{k}\right)^{p}=t^{-p v(1-n)}>1 .
$$

The final inequality follows from $-p v(1-n)>0$.

(c) $\mu^{q s-k} \lambda^{p s}>1$, because $s$ is chosen so that $(q s-k) / p s>r$.

(d) $t^{p v-v n}>1$, because $p v-v n>0$. 
Therefore, in this case we may choose our sequence of rationals to be

$$
r_{i}=\frac{i+p q(v+s)}{v+s}
$$

for $i \geq 0$, as the corresponding elements $\mu_{C}^{i+p q(v+s)} \lambda_{C}^{v+s}$ are positive in the leftordering for $i \geq 0$.

\section{Surgery on satellites}

Let $T$ denote the solid torus containing a knot $K^{P}$, we require that $K^{P}$ is not contained in any three-ball inside $T$. The knot $K^{P}$ will be called the pattern knot. Let $K^{C}$ denote a knot in $S^{3}, K^{C}$ will be called the companion knot. We construct the satellite knot $K$ with pattern $K^{P}$ and companion $K^{C}$ as follows.

Let $h: \partial T \rightarrow \partial\left(S^{3} \backslash \nu\left(K^{C}\right)\right)$ denote a diffeomorphism from the boundary of $T$ to the boundary of the complement of $\nu\left(K^{C}\right)$, which carries the longitude of $\partial T$ onto the longitude of the knot $K^{C}$. The knot $K$ is then realized as the image of the knot $K^{P}$ in the manifold

$$
S^{3} \backslash \nu\left(K^{C}\right) \sqcup_{h} T=S^{3} .
$$

Lemma 4.1. [19, Proposition 3.4] There exists a homomorphism $\phi: \pi_{1}(K) \rightarrow \pi_{1}\left(K^{P}\right)$ that perserves peripheral structure.

Proof. We can compute the fundamental group $\pi_{1}(K)$ by using the Seifert-Van Kampen theorem. Since

$$
S^{3} \backslash \nu(K)=S^{3} \backslash \nu\left(K^{C}\right) \sqcup_{h} T \backslash \nu\left(K^{P}\right),
$$

the group $\pi_{1}(K)$ is the free product $\pi_{1}\left(K^{C}\right) * \pi_{1}\left(T \backslash \nu\left(K^{P}\right)\right)$, with amalgamation as follows: the meridian of $K^{C}$ is identified with the meridian of $T$, and the longitude of $K^{C}$ is identified with the longitude of $T$.

Let $N$ denote the normal closure in $\pi_{1}(K)$ of the commutator subgroup of $\pi_{1}\left(K^{C}\right)$. The quotient $\pi_{1}(K) / N$ can be considered as the result of killing the longitude of $T$. Topologically we can think of this quotient as gluing a second solid torus $T^{\prime}$ to the torus $T$ containing $K^{P}$, in such a way that the meridian of $T^{\prime}$ is glued to the longitude of $T$. The result is that $\pi_{1}\left(K^{C}\right)$ collapses to a single infinite cyclic subgroup, and the group $\pi_{1}(K) / N$ is isomorphic to $\pi_{1}\left(K^{P}\right)$. The desired homomorphism $\phi$ is the quotient map $\pi_{1}(K) \rightarrow \pi_{1}(K) / N$.

Proposition 4.1. Suppose that $K$ is a satellite knot with pattern knot $K^{P}$, and $r \in \mathbb{Q}$ is any rational number. If $\pi_{1}\left(S_{r}^{3}\left(K^{P}\right)\right)$ is left-orderable and $S_{r}^{3}(K)$ is irreducible, then $\pi_{1}\left(S_{r}^{3}(K)\right)$ is left-orderable.

Proof. By Lemma 4.1, there exists a homomorphism $\phi: \pi_{1}(K) \rightarrow \pi_{1}\left(K^{P}\right)$ that preserves peripheral structure, so there exists an induced map

$$
\phi_{r}: \pi_{1}\left(S_{r}^{3}(K)\right) \rightarrow \pi_{1}\left(S_{r}^{3}\left(K^{P}\right)\right),
$$

for every $r \in \mathbb{Q}$. Whenever $\pi_{1}\left(S_{r}^{3}\left(K^{P}\right)\right)$ is left-orderable (and hence non-trivial, since our definition of left-orderable does not allow $\mathcal{P}=\emptyset$ ) the image of $\phi_{r}$ is non-trivial and $\pi_{1}\left(S_{r}^{3}(K)\right)$ is left-orderable [2, Theorem 1.1] . 
Proof of Theorem 1.4. By [7], $p q$-surgery on a $(p, q)$-cable knot yields a reducible manifold. Since the minimal geometric intersection number between reducible slopes is \pm 1 [8], $r$-surgery on a $(p, q)$-cable yields an irreducible manifold whenever $r<p q-p-q$. Moreover, a $(p, q)$-cable knot can be described as a satellite knot with pattern knot $T_{p, q}$, the $(p, q)$-torus knot. Therefore, for $r<p q-p-q$ we can apply Proposition 4.1 to conclude that $\pi_{1}\left(S_{r}^{3}(K)\right)$ will be left-orderable whenever $\pi_{1}\left(S_{r}^{3}\left(T_{p, q}\right)\right)$ is left-orderable.

We may now combine known results for surgery on torus knots in this setting. On the one hand, $\pi_{1}\left(S_{r}^{3}\left(T_{p, q}\right)\right)$ is an L-space whenever $r \geq 2 g-1$ [14, Proposition 9.5] (see in particular [9, Lemma 2.13]), where $g=g\left(T_{p, q}\right)$ is the Seifert genus given by $g\left(T_{p, q}\right)=\frac{1}{2}(p-1)(q-1)$. On the other, since $S_{r}^{3}\left(T_{p, q}\right)$ is Seifert fibred or a connect sum of lens spaces for every $r$ [13], $S_{r}^{3}\left(T_{p, q}\right)$ is an L-space if and only if $\pi_{1}\left(S_{r}^{3}\left(T_{p, q}\right)\right)$ is not left-orderable [1] (see also [16, 21]). In particular, $\pi_{1}\left(S_{r}^{3}\left(T_{p, q}\right)\right.$ ) is left-orderable whenever $r$ is less than $2 g\left(T_{p, q}\right)-1$ and the result follows.

\section{Acknowledgments}

We thank Josh Greene, Tye Lidman and Dale Rolfsen for helpful comments on an earlier draft of this paper. Both authors partially supported by NSERC postdoctoral fellowships.

\section{References}

[1] S. Boyer, C. McA. Gordon and L. Watson, On L-spaces and left-orderable fundamental groups, preprint, arXiv:1107.5016.

[2] S. Boyer, D. Rolfsen and B. Wiest, Orderable 3-manifold groups, Ann. Inst. Fourier (Grenoble) 55(1) (2005), 243-288.

[3] D. Calegari and N. M. Dunfield, Laminations and groups of homeomorphisms of the circle, Invent. Math. 152(1) (2003), 149-204.

[4] A. Clay, The space of left orderings of a group with applications to topology, Ph.D. thesis, University of British Columbia, 2010.

[5] A. Clay and D. Rolfsen, Ordered groups, eigenvalues, knots, surgery and L-spaces. Math. Proc. Cambridge Philos. Soc., 152(01) (2012), 115-129.

[6] A. Clay and L. Watson, Left-orderable fundamental groups and Dehn surgery, preprint, arXiv: 1009.4176.

[7] C. McA. Gordon, Dehn surgery and satellite knots, Trans. Amer. Math. Soc. 275(2) (1983), $687-708$.

[8] C. McA. Gordon and J. Luecke, Reducible manifolds and Dehn surgery, Topology 35(2) (1996), 385-409.

[9] M. Hedden, On knot Floer homology and cabling: 2, Int. Math. Res. Not. IMRN, (12) (2009), $2248-2274$.

[10] J. Hom, A note on cabling and L-space surgeries, Algebr. Geom. Topol. 11(1) (2011), 219-223.

[11] J. Howie and H. Short, The band-sum problem, J. London Math. Soc. (2) 31(3) (1985), 571-576.

[12] V. M. Kopytov and N. Ya. Medvedev, Right-ordered groups, Siberian School of Algebra and Logic. Consultants Bureau, New York, 1996.

[13] L. Moser, Elementary surgery along a torus knot, Pacific J. Math. 38 (1971), 737-745.

[14] P. Ozsváth and Z. Szabó, Knot Floer homology and rational surgeries, preprint, arXiv:0504404.

[15] P. Ozsváth and Z. Szabó, On knot Floer homology and lens space surgeries, Topology 44(6) (2005), 1281-1300.

[16] T. Peters, On L-spaces and non left-orderable 3-manifold groups, preprint, arXiv:0903.4495.

[17] R. Roberts, J. Shareshian and M. Stein, Infinitely many hyperbolic 3-manifolds which contain no Reebless foliation, J. Amer. Math. Soc. 16(3) (2003), 639-679 (electronic). 
[18] A. S. Sikora, Topology on the spaces of orderings of groups, Bull. London Math. Soc. 36(4) (2004), 519-526.

[19] D. S. Silver and W. Whitten, Knot group epimorphisms, J. Knot Theory Ramifications, 15(2) (2006), 153-166.

[20] C. M. Tsau, Isomorphisms and peripheral structure of knot groups, Math. Ann. 282(2) (1988), $343-348$.

[21] L. Watson, Involutions on 3-manifolds and Khovanov homology, Ph.D. thesis, Université du Québec à Montréal, 2009.

Cirget, Université du Québec À Montréal, Case postale 8888, Succursale Centreville, Montréal QC, H3C 3P8, Canada

E-mail address: aclay@cirget.ca

$U R L:$ http://thales.math.uqam.ca/ aclay

Department of Mathematics, UClA, 520 Portola Plaza, Los Angeles, CA 90095, USA

E-mail address: lwatson@math.ucla.edu

URL: http://www. math.ucla.edu/ l watson 
